\title{
Los medios distractores en el aula de clase
}

\author{
Arlen Meryfel Picado Juárez \\ Universidad Nacional Autónoma de Nicaragua, Managua/FAREM-Estelí \\ arlenmery@yahoo.com \\ Darwing Joel Valenzuela Flores \\ Universidad Nacional Autónoma de Nicaragua, Managua /FAREM-Estelí. \\ dvalenzuela337@gmail.com \\ Yirley Indira Peralta Calderón
}

Universidad Nacional Autónoma de Nicaragua, Managua /FAREM-Estelí.

indiraperalta25@yahoo.es

Fecha de recibido: 6 de abril de $2015 \quad$ Fecha de aprobado: 17 de julio de 2015

\section{RESUMEN}

En la actualidad la tecnología avanza rápidamente, tiempo donde es considerada como la revolucionaria de las comunicaciones. Esto vino a cambiar la velocidad del intercambio de información en los diferentes ámbitos de la vida laboral, familiar y educativa, en este último, la tecnología ha afectado significativamente los procesos de enseñanza aprendizaje, hasta llegar a convertirse en uno de los principales distractores en el aula de clase.. La conducta del estudiantado ha cambiado, al igual que la concentración en sus estudios durante el desarrollo de la clase, ambos elementos han sido identificados en los estudiantes de la carrera de Administración de Empresas del turno diurno, es por ellos que resulta interesante realizar este estudio, para conocer más a fondo las razones de estos cambios. Los docentes tienen el reto de prestar atención para afrontar y sacarle provecho a los dispositivos móviles que utilizan los estudiantes durante los procesos de la enseñanza aprendizaje.

Palabras claves: dispositivo móvil, aula de clase, medios distractores.

\section{ABSTRAC}

Currently technology is advancing rapidly, time where is being considered revolutionary of communications. This came to change the speed of exchange of information in different fields of work, family and educational life in this last aspect technology has significantly affected the teaching and learning processes, up to becoming one of the main distractions in classroom. The student's behavior has changed, as their concentration in during the development of the class, both elements have been identified in students of the career of Business Administration from the daytime shift, it is for them that this study is interesting, to be able to learn more about the reasons for these changes. Teachers have the challenge of paying attention to face and take advantage of mobile devices used by students during the learning process.

Keywords: mobile device, classroom, distracting media. 


\section{INTRDUCCIÓN}

Nicaragua es un país considerado en vías de desarrollo, donde una de las preocupaciones más importantes del gobierno es el fortalecimiento de la educación, hoy en día el acceso a ella es de carácter gratuito. La educación superior ha crecido en gran medida debido al fácil acceso a la tecnología y grandes porcentajes de retención escolar, lo que cada año se desencadena en mayor demanda de las universidades públicas. Sin embargo, la vida estudiantil del universitario ha venido avanzando al mismo ritmo que avanza la tecnología, pues la facilidad de mantenerse comunicado con amigos y familiares se ha convertido en una necesidad que debe ser satisfecha, para lo cual se han creado un sin número de dispositivos que facilitan y satisfacen este tipo de demanda, como los celulares, tablets y computadoras portátiles, que permiten la conexión a las diferentes redes sociales, las que poseen aplicaciones que compensan gustos de clientes exigentes.

Los dispositivos tecnológicos poseen diversos usos, que si se le da el uso adecuado, pueden facilitar los procesos de enseñanza aprendizaje en las aulas universitarias. No obstante, la efectividad de los procesos de enseñanza aprendizaje se han visto amenazados por la tecnología misma y las condiciones en que se desarrollan. En ese contexto, consideramos investigar ¿Cuáles son los medios distractores presentes en los salones de clases de la Facultad Regional Multidisciplinaria, Estelí? Ésta investigación es considerada de mucha importancia, pues contestar esta interrogante permitirá formular las medidas que contrarrestarán los efectos negativos de los medios distractores, permitiendo de esta manera lograr los objetivos académicos propuestos para el desarrollo de las asignaturas.

\section{DESARROLLO}

El principal interés de un estudiante debe ser la adquisición de conocimiento, aprender y prestar atención a la explicación del docente. A través de nuestra experiencia docente, hemos observado que muchos estudiantes no muestran interés en sus aprendizajes y caen en la distracción, que no es más que la desviación de la atención por parte de alguien cuando éste debe de atender algo.

En ese sentido, la tecnología es una de las causas principales de la distracción de los alumnos y es difícil convencerlos de las consecuencias que conlleva el uso excesivo de un 
dispositivo de mano en su vida académica. Sin embargo, la tecnología no es del todo mala si se sabe administrar correctamente el tiempo de uso, contribuye en la vida diaria en el quehacer de tareas, búsqueda de información e incluso fortalece lazos familiares.

Existen una serie de estímulos ambientales que puede influir de forma negativa en la concentración del estudio, estos pueden ser elementos que se utilicen en el momento y que estén relacionados con el mismo, convirtiéndose en distractores; como gestos o pequeñas conductas que hacen perder un tiempo valioso. Los estudiantes tienen pequeñas estrategias" que utilizan para distraerse, logrando sorprender con cosas nuevas constantemente, no se conocen todas. Para los autores Abad Figueroa, Palomec Cabrera, Toscano Gutiérrez (2013) los distractores del aprendizaje son:

Cualquier situación u objeto que atrae la atención alejándola de las tareas de estudio. Por ejemplo: ruidos, voces, fotos, personas, música, etc. En general todo lo que a nuestro alrededor pueda llamarnos la atención. Por otra parte, la atención es selectiva y se fija en aquello que nos motiva. Para mejorar la atención hay que trabajar en un entorno adecuado y libre de estímulos que llamen la atención.

Una queja o comentario recurrente de los profesores de aula se refiere a la distracción de sus alumnos con la tecnología: en lugar de centrarse en la clase misma con una aplicación educativa como núcleo de una clase están redactando mensajes de texto, navegando, tuteando, etc. En la medida en que empezamos a poner restricciones, prohibiciones, concluyen los profesores surgen los conflictos. (pág. 2)

A continuación se detallan los distractores más comunes:

- Reproductores de música: La escucha de música es poco recomendable a la hora de rendir satisfactoriamente en el estudio.

- Carteles, póster o fotos: En la mayoría de las aulas de clases existe este tipo de materiales, por lo que puede ser un factor que puede interrumpir la concentración del estudiante, si no es posible su eliminación, se aconseja trasladarlos a lugares menos visibles de donde se encuentre el estudiante.

- Ventana: La ventana por sí misma no es un elemento distractor, pero sí lo puede ser, cuando se convierte en el sustituto natural del celular para intentar distraernos y lograr pasar de la forma más entretenida posible horas de estudio; para esto se 
sugiere cerrar las cortinas o la persiana, aunque ello implique utilizar luz artificial o, en último caso, intentar modificar el lugar de estudio dentro de la misma aula de clase.

- Teléfono móvil: Por este medio, los estudiantes satisfacen la necesidad de estar constantemente "conectados" a sus amigos a través de llamadas, mensajes o redes sociales; si esto se produce durante el tiempo de estudio, se convierte en un elemento altamente distractor.

Este último elemento distractor, es el que los estudiantes usan frecuentemente en los salones de clase de FAREM Estelí. Desde nuestra experiencia con los grupos de tercero y cuarto año de la carrera de Administración de Empresas, turno diurno, son muestra de éste fenómeno que interviene en los procesos de enseñanza aprendizaje. Es así que, las redes sociales, la comunicación a través de llamadas y mensajería interrumpen, el proceso de aprendizaje, ya que generan llamados de atención, por encontrarse prohibido su uso durante las clases. Al respecto, Jarquín (2014) expresa que entre los universitarios de Centroamérica, los nicaragüenses son los que más usan las redes sociales.

Los universitarios son los principales usuarios de redes sociales en Nicaragua y la preferida es Facebook, pero no aprovechan todo el potencial de esos servicios, consideraron especialistas consultados por El Nuevo Diario, con base en un estudio regional y una investigación local. El estudio "Redes Sociales en Centroamérica 2013", de la firma iLifebelt, una empresa especializada en mercadeo en línea, muestra que en la región el $40 \%$ de los usuarios de redes sociales son estudiantes universitarios.

La experiencia desagradable de algunos docentes que comparten con los grupos inmersos con esta temática, impulsó una encuesta de opinión a los estudiantes para conocer las razones por las cuales hacen uso frecuente del dispositivo en el momento que se desarrolla la clase, contando con una muestra representativa de 55 estudiantes, el 22\% afirma que si hace uso del dispositivo cuando el docente está facilitando el proceso de enseñanza aprendizaje. Sin embargo, el 76\% de los estudiantes está consciente que el hacer uso del celular interfiere en los aprendizajes durante la clase, generando pérdida de concentración, interrupción a los demás compañeros y falta de respeto al maestro. El 24\% de los encuestados 
menciona que no interfiere en el proceso de enseñanza aprendizaje, justificando que depende en gran medida de la concentración y habilidad mental que posean, consideran que no interrumpen la clase porque únicamente revisan la notificación o mensaje y continúan atendiendo a la clase y contestan los mensajes de forma rápida.

Otro aspecto investigado con los estudiantes, es su opinión sobre la prohibición del uso de celulares dentro de los salones de clase, donde el $42 \%$ manifiesta estar de acuerdo con la prohibición del mismo, pues considera que es un mal hábito adquirido, convirtiéndose en un medio de distracción para los estudiantes, afectando la apropiación de conocimientos. Otros opinan que prohibir el celular es una manera de forzar al estudiante a estar atento a la clase, permitiendo una mejor armonía y concentración en el aula de clases y por consiguiente la integración de los estudiantes en los procesos de enseñanza aprendizaje. El 47\% expresa no estar de acuerdo con la medida restrictiva, pues consideran que éste debe usarse para llamadas de emergencia, que no todas las personas lo utilizan para interrumpir las clases. Por otro lado, un $11 \%$ se encuentra indeciso, pues no lo consideran una interferencia y que podría promoverse el uso adecuado y aprovechar el recurso como una herramienta didáctica.

Es importante mencionar que no sólo los móviles generan distracción en los procesos educativos, también, es meritorio aclarar que las condiciones de los salones de clase dan pie para la intervención de otros distractores. Como bien se sabe, el aula de clases es un salón, en un edificio que se destina a actividades de enseñanza aprendizaje, utilizado en la educación, donde el estudiante esta interactuando con sus compañeros. Intercambian sus experiencias y conocimientos alrededor de las vivencias cotidianas, también son participes de la construcción de nuevos aprendizajes basados en un modelo educativo.

EcuRed (2011) define el aula de clases de la siguiente manera:

Es generalmente un salón de dimensiones variables que debe contar con espacio suficiente como para albergar a los sujetos que intervienen en el proceso de enseñanza-aprendizaje: el docente y los alumnos. Este espacio consta normalmente de un área para el trabajo del educador y con un área más amplia donde trabajan los alumnos de la manera más cómoda posible a fin de obtener los mejores resultados. (párr.1) 
La implementación del aula como espacio esencial para el desarrollo del proceso de enseñanza-aprendizaje tuvo lugar especialmente en el siglo XIX, momento en el cual la educación dejó de estar en manos de unos pocos y comenzó lentamente a extenderse a todos los sectores de la sociedad. Las aulas son entonces habitaciones o salas de una institución mayor en las que un grupo específicamente determinado de alumnos asiste a la clase del profesor, pudiendo esta situación repetirse en todos los niveles escolares desde el inicial hasta el universitario. Ciertas disciplinas educativas requieren contar con espacios específicos, entre ellas las aulas de computación, aulas de música, aulas de educación física y aulas de idiomas o laboratorios.

\section{Características del aula adecuada}

Las aulas de clases deben poseer un sin número de características que permitan o faciliten el desarrollo de las estrategias activas participativas de enseñanzas aprendizajes, EcuRed (2011) hace mención de algunas de esas características:

- Ubicación de las sillas: Estás deben de estar en dirección hacia el docente, donde hará su exposición, otra forma es en círculos, favoreciendo así el contacto con los integrantes, la comunicación espontanea, cómoda y permanente.

- Comodidades básicas: El aula de clases debe contener luz, limpieza, espacio, ventilación y una adecuada temperatura; estos son componentes claves que brindan comodidad y tranquilidad al impartir clases, tanto para el docente como para el estudiante. Éste debe ser un ambiente estimulante, limpio y ordenado para que transmita seguridad al estudiante en proceso de aprendizaje.

- Reglas de conducta: Estas propiciaran las mejores condiciones de estudio y trabajo, también el respeto entre los estudiantes y docentes.

- La decoración: Esta es considerada como un aspecto motivante en el aprendizaje, en el transcurso de las clases los estudiantes podrán elaborar murales y otros materiales que decoraran y ambientaran el salón de clases. Hay que prestar atención a esto, ya que no es conveniente exagerar, el estudiante suele distraerse fácilmente y también podría ocasionar desmotivación; un ambiente cargado puede llegar a generarles estrés. 
- La acústica: las adecuadas condiciones del sonido facilitaran una correcta escucha y concentración del estudiante, también hay que tomar en cuenta el sonido externo al salón de clases, este puede ser un distractor durante el proceso de enseñanza aprendizaje.

Los elementos antes mencionados, permitirán tener un ambiente propicio para el desarrollo de las diferentes actividades en el proceso de enseñanza aprendizaje, si uno o varios de ellos llega a faltar puede ocasionar desmotivación, falta de concentración, hasta la deserción estudiantil. Es tarea de los directivos de los centros educativos facilitar un ambiente apropiado con óptimas condiciones, las que facilitarán el desarrollo y culminación del proceso de enseñanza aprendizaje del educando en un determinado periodo.

El grupo de tercer año de la carrera de Administración de Empresas está situado en el aula con numeración 606, ubicada en la planta baja del pabellón Priscila Fuentes ${ }^{1}$ el que cuenta con dimensiones de 6x5 metros aproximadamente. Posee dos puertas de acceso, una delantera y otra trasera. En su interior, cuenta con cuarenta pupitres, mesa y una silla para el docente, así como pizarra acrílica adherida a la pared. Los ventanales están situados en el lado oeste del salón, las que se adornan con cortinas de tono rojo quemado y son extendidas únicamente si los rayos del sol penetran con mucha claridad, por lo general, éstas se mantienen cerradas para evitar el acceso de polvo y se abren si las condiciones climáticas ameritan tener una mayor circulación de aire que refresque el ambiente. Físicamente los salones poseen condiciones aceptables, pues los pupitres son cómodos y pueden manipularse conforme lo requiera el docente, ya sean en hileras, semicírculos alrededor del salón o para pequeños círculos que conforman los equipos de trabajo.

Dentro de las comodidades básicas, los salones poseen las instalaciones correspondientes de energía eléctricas y las lámparas están dispuestas de manera equitativa en toda el aula de clase, las que no son utilizadas por ese grupo, debido a que pertenecen al turno matutino y aprovechan la claridad del día. Dentro del salón se encuentra un recipiente para colocar basura generada durante los procesos de enseñanza aprendizaje. La Facultad cuenta con personal encargado de la limpieza. Para este pabellón hay un conserje asignado. La decoración de las aulas de clase es simple, las paredes están matizadas en tono blanco

\footnotetext{
${ }^{1}$ Todos los pabellones de la facultad tienen nombres de Héroes y Mártires y personajes importantes en el
} quehacer educativo de la universidad. 
hueso con puertas de madera tono rojo quemado y la pared del lado sur del salón es utilizadas por los grupos asignados al mismo, para la exposición de murales resultantes de los procesos de enseñanzas aprendizajes, no considerados como distractores, por encontrarse a espaldas de los estudiantes.

Partiendo de nuestra experiencia y observación en el grupo de clase, consideramos que la acústica del salón es uno de los distractores con mayor presencia, ya que, cerca del pabellón hay un cafetín al que acuden un número considerable de estudiantes. A pocos metros, se encuentran las canchas deportivas, a las que acuden estudiantes a practicar diversidad de disciplinas deportivas durante todo el día y parte de la noche, por lo que la última característica referente a las normas de conducta se torna un poco más exigente debido al esfuerzo de concentración que debe ser más agudo y la resistencia a la tentación de salir del salón debe ser más prorrogable.

Por otro lado, para el grupo de cuarto año de la carrera, las condiciones no difieren mucho de lo anteriormente expuesto, pues ellos se sitúan en el aula 607 del mismo pabellón, a diferencia que éste está situado en la segunda planta y los estudiantes pertenecen al turno nocturno, lo que implica el uso indispensable de las lámparas para poder iluminarse, acción que conlleva a la intervención de un distractor no tomado en cuenta en la teoría, como son los insectos o bichos atraídos por la iluminación, se convierten en otra más, por las condiciones geoclimáticas donde se ubica la Facultad.

\section{CONCLUSIONES}

Al finalizar nuestro estudio, hemos llegado a la conclusión que los teléfonos móviles son el principal medio distractor en los salones de clases de los grupos de tercer y cuarto año de Administración de Empresas, del turno diurno de la FAREM-Estelí. Los distractores presentes en el salón de clase, así como los factores externos, conllevan a la perdida de interés en el estudio, se exterioriza una mayor desmotivación e incluso la deserción estudiantil.

En este estudio, una parte significativa afirma que hacen uso del dispositivo cuando el docente está facilitando el proceso de enseñanza aprendizaje, sin embargo, la mayoría de los estudiantes están conscientes de que el uso del mismo, interfiere en los aprendizajes durante la clase. Expresan que provoca la pérdida de concentración, interrupción a los demás 
compañeros y también falta de respeto al maestro. A los estudiantes se les hace difícil no utilizar este medio distractor durante la clase, a pesar de que es considerando su uso como un mal hábito o adicción.

Como docentes, debemos de encontrar alternativas para utilizar este medio distractor como una herramienta, que facilite el proceso de aprendizaje, de esta manera se obtendrán varios beneficios, entre ellos: facilitar que los alumnos estén atentos en las diferentes actividades y por tanto se puedan reforzar los objetivos a conseguir, aumento en la participación por parte de los estudiantes, lo que propiciará el desarrollo de iniciativas, dado que las actividades llevan consigo la constante toma de decisiones ante las respuestas del dispositivo móvil a sus acciones.

Otro aspecto positivo es la interacción constante, que propicia el trabajo individualizado y creativo durante los procesos de enseñanza aprendizaje. Es conveniente mencionar que los docentes deben evitar caer en la dependencia tecnológica y no olvidar que las diferentes formas de aprendizaje de un estudiante a otro varían. Si no estamos atentos a estos aspectos, puede generarse un efecto negativo en el acercamiento a las tecnologías y al interés de la temática que aborda el docente.

\section{REFERENCIAS}

Abad Figueroa, C., Palomec Cabrera, J. A., y Toscano Gutiérrez, J. F. (2013). Distractores que intervienen en el aprendizaje del adolescente en la escuela secundaria. Obtenido de Academia de Ciencias de Morelos, A.C: http://www.acmor.org.mx/reportescongreso/2013/secundaria/sociales/1008distractores-que.pdf

EcuRed. Connocimiento con todos y para todos. (2011). EcuRed. Connocimiento con todos y para todos. Recuperado el 2 de marzo de 2015, de EcuRed. Connocimiento con todos y para todos: http://www.ecured.cu/index.php/Aula

Jarquín, L. (23 de Junio de 2014). Universitarios nicas, adictos a las redes sociales. El Nuevo Diario. Obtenido de El nuevo diarios, Managua-Nicaragua: http://www.elnuevodiario.com.ni/especiales/323025-universitarios-nicas-adictos-aredes-sociales 